\title{
Pain modulation is affected differently in medication-overuse headache and chronic myofascial pain - A multimodal MRI study.
}

Michels $L^{1}$, Christidi $F^{1}$, Steiger $V R^{2}$, Sandor $P S^{3,4}$, Gantenbein $A R^{3,4}$, Landmann $G^{5}$, Schreglmann $S R^{6}$, Kollias $S^{1}$, Riederer $F^{4,7}$

${ }^{1}$ Clinic of Neuroradiology, University Hospital Zurich, Zurich, Switzerland

${ }^{2}$ Division of Neuropsychology, Institute of Psychology, University of Zurich, Zurich, Switzerland

${ }^{3}$ RehaClinic, Bad Zurzach \& Baden, Switzerland

${ }^{4}$ University of Zurich, Zürich, Switzerland

${ }^{5}$ Centre for Pain Medicine, Swiss Paraplegic-Centre, Nottwil, Switzerland

${ }^{6}$ Department of Neurology, University Hospital Zurich, Switzerland

${ }^{7}$ Neurological Center Rosenhuegel and Karl Landsteiner Institute for Epilepsy Research and Cognitive Neurology, Vienna, Austria

Corresponding author: Lars Michels, lars.michels@usz.ch

Number of figures: 6 , Number of tables: 5

Keywords: functional magnetic resonance imaging, functional connectivity, medication overuse, headache, diffusion tensor imaging, myofascial pain. 


\begin{abstract}
Background: Neuroimaging studies revealed structural and functional changes in medication-overuse headache $(\mathrm{MOH})$, but it remains unclear whether similar changes could be observed in other chronic pain disorders.
\end{abstract}

Methods: In this cross-sectional study, we investigated functional connectivity (FC) with resting state functional magnetic resonance imaging (fMRI) and white matter integrity using diffusion tensor imaging (DTI) to measure fractional anisotropy (FA) and mean diffusivity $(\mathrm{MD})$ in patients with $\mathrm{MOH}(\mathrm{N}=12)$ relative to two control groups: patients with chronic myofascial pain $(\mathrm{N}=11)$ and healthy controls $(\mathrm{CN} ; \mathrm{N}=16)$.

Results: In a data-driven approach we found hypoconnectivity in the fronto-parietal attention network in both pain groups relative to $\mathrm{CN}$ (i.e. $\mathrm{MOH} \angle \mathrm{CN}$ and $\mathrm{MYO}<\mathrm{CN}$ ). In contrast, hyperconnectivity in the saliency network (SN) was detected only in $\mathrm{MOH}$, which correlated with FA in the insula. In a seed-based analysis we investigated FC between the periaqueductal grey (PAG) and all other brain regions. In addition to overlapping hyperconnectivity seen in patient groups (relative to $\mathrm{CN}$ ), $\mathrm{MOH}$ had a distinct connectivity pattern with lower FC to parieto-occipital regions and higher FC to orbitofrontal regions compared to controls. FA and MD abnormalities were mostly observed in $\mathrm{MOH}$, involving the insula.

Conclusions: Hyperconnectivity within the SN along with associated white matter changes therein suggest a particular role of this network in $\mathrm{MOH}$. In addition, abnormal connectivity between the PAG and other pain modulatory (frontal) regions in $\mathrm{MOH}$ are consistent with dysfunctional central pain control.

\title{
Introduction
}


Medication-overuse headache $(\mathrm{MOH})$ affects $1-2 \%$ of the general population and causes significant social and financial burden $(1,2)$. $\mathrm{MOH}$ is defined as headache that develops or significantly worsens during overuse of acute pain medication (3). During medication overuse, pain thresholds in cephalic and extracephalic regions were found to be reduced in animal and human studies, probably because of drug-induced latent sensitization of the trigeminovascular system $(4,5)$, which was reversible after detoxification. Alterations in central modulation of trigeminal nociceptive input have been proposed in the pathophysiology of $\mathrm{MOH}$. These may involve an imbalance within descending pain modulating systems towards pain facilitation (6-8).

Metabolic and structural alterations have been described in $\mathrm{MOH}$, including hypometabolism in the orbitofrontal cortex (OFC) in a PET-study (9) and grey matter (GM) increases in the striatum, thalamus and the midbrain (10). After detoxification the GM increase in the midbrain including the periaqueductal grey (PAG), which is involved in descending pain modulation, was reversible in patients who improved clinically, whereas other GM changes were not (11). Hypometabolism in the OFC persisted after detoxification, whereas other hypometabolic regions such as thalamus and ventral striatum/insula returned to normal. Persistent structural GM changes and hypometabolism in OFC after detoxification have been related to patients' 
dependence on the analgesic compound or predisposition to medication overuse relapse $(9,11)$.

It is assumed that the human brain is highly active in the absence of explicit input or output (12). Resting state functional magnetic resonance imaging (RS-fMRI) examines spontaneous low frequency fluctuations in the blood oxygenation level dependent (BOLD) signal in absence of external tasks or stimuli and can provide a measure of functional connectivity (FC) between various brain regions (13) and networks (14). Resting-state networks, i.e. spatially distinct areas of the brain that show synchronous BOLD signal fluctuations at rest, have been consistently identified using various methodological approaches $(14,15)$, including the default-mode network (DMN), fronto-parietal attention network (FPN), sensorimotor network (SMN), or saliency network (SN), which comprised of the bilateral insulae and dorsal anterior cingulate cortex (ACC). One study has examined resting state $\mathrm{FC}$ in $\mathrm{MOH}$ and reported decreased FC between the precuneus and fronto-parietal regions within the DMN and increased FC between precuneus and hippocampal/temporal areas (16). Increased FC between the PAG and both nociceptive and somatosensory regions was found in patients with episodic migraine between the attacks (17). Attack frequency correlated negatively with FC between the PAG and prefrontal regions, anterior cingulate cortex and thalamus, probably reflecting impaired pain modulation. The PAG is functionally and 
anatomically connected with forebrain structures involved in pain processing $(18,19)$ and is probably involved in the pathophysiology of migraine and $\mathrm{MOH}(11,17,20)$.

White matter (WM) changes have been documented in migraine and other chronic pain conditions. These microstructural alterations are inferred from diffusion tensor imaging (DTI) indices, such as fractional anisotropy (FA), which quantifies the degree of preferential restriction in water diffusion, and mean diffusivity (MD), which measures the overall magnitude of water diffusion (21). Patients with episodic migraine showed lower FA in the thalamocortical tract, trigeminothalamic tract and (in patients without aura) ventrolateral PAG (22), as well as reduced FA in the visual-motion processing network (23). Yet, whole-brain WM alterations have not been investigated in patients with $\mathrm{MOH}$.

In the present study we performed a multi modal imaging approach to investigate FC and WM changes in patients with $\mathrm{MOH}$ compared to healthy controls (CN), as functional and structural changes are probably interrelated. A group of patients with chronic myofascial pain (MYO) without evidence for comorbid $\mathrm{MOH}$ was also studied as a second control group, to investigate which changes might be attributable to chronic pain rather than specifically to $\mathrm{MOH}$. Myofascial pain is a common widespread musculoskeletal pain syndrome that is thought to be associated with central 
sensitization (26). In myofascial pain, e.g. fibromyalgia, FC differences have been described within the SN and FPN $(24,25)$. For the FC analysis, we applied a data-driven approach on the network level: the independent component analysis (ICA). We hypothesized altered $\mathrm{FC}$ in the $\mathrm{SN}$ of $\mathrm{MOH}$ and $\mathrm{MYO}$ and hypoconnectivity in the FPN in $\mathrm{MOH}$ compared to controls, based on previous studies $(24,25,27)$. Next, we performed a seed-based analysis to investigate correlations between the spontaneously fluctuating BOLD signal in the seed region (PAG) and all other brain regions, similar as in (17). We hypothesized altered FC to somatosensory regions in $\mathrm{MOH}$. Functional hyperconnectivity indicates a stronger coupling within a neural network or between a seed region and the rest of the brain in one than in the other group, as a result of more similar spontaneously fluctuating $\mathrm{fMRI}$ signal time courses. WM changes were examined on the whole-brain level using tract based spatial statistics (TBSS), and local between-group WM differences were further explored and linked to clinical symptom scores. TBSS provides measures of FA and MD to determine deviations in WM (microstructural) integrity and membrane density, respectively. We hypothesized to find altered WM integrity in frontal brain regions, such as the OFC and insular cortex, as it is known that these regions show structural changes in $\mathrm{MOH}(10)$. 


\section{Material and Methods}

\section{Participants}

The study was approved by the ethics committee of the Canton Zurich. Participants gave written and informed consent. The present study included 12 patients with $\mathrm{MOH}$, evolving from episodic migraine, according to diagnostic criteria of the International Headache Society (3) (mean age: 44.4 range: 31-65 years, 9 women), 16 healthy controls (CN, mean age: 42.4, range: 31-61 years, 7 women), free from any pain, and 11 patients with chronic MYO (mean age: 38.3 , range: $24-56$ years, 8 women). Subjects did not differ with respect to age and sex. A list with the full demographics and clinical data is provided in Table 1.

\section{Clinical assessment}

Participants were excluded if they had evidence of structural lesions in the MRI, cardiovascular disease, any signs of neurological disease -other than the studied disorder or severe psychiatric disorders-, and standard contradictions for MRI. All MYO patients were evaluated in a multidisciplinary setting involving neurologists, psychologists and orthopaedists. The diagnosis of MYO was established according to 
published clinical criteria (26). All patients with MYO had normal nerve conduction studies, somatosensory evoked potentials and thermography. Inflammatory rheumatologic disease was excluded by laboratory tests. In all participants depression and anxiety were assessed using the Hospital Anxiety and Depression Scale (HADS) questionnaire (28). Pain intensity during scan was assessed using a visual analogue scale (VAS), ranging from 0 (no pain) to 10 (strongest pain). Duration of pain disorder (years) and headache days per month in $\mathrm{MOH}$ were also recorded.

\section{Functional imaging}

Whole-brain resting-state $\mathrm{fMRI}$ data were acquired with a $\mathrm{T} 2{ }^{*}$-weighted echo planar imaging (EPI) single-shot sequence and an eight-channel head coil. The following recording parameters were used: TR/TE: $2 \mathrm{~s} / 35 \mathrm{~ms}$, EPI factor: 53, flip angle: $78^{\circ}$, number of slices: 30 , voxel resolution: $3 \times 3 \times 4 \mathrm{~mm}$, scan order: ascending, scan duration: 6:30 minutes. MR-compatible headphones and cushions were used to minimize subjects' head motion. Participants were instructed to lie still, fixate on a white cross on dark background, to be relaxed, but to remain awake. 
FMRI pre-processing

Pre-processing and statistical analysis of the fMRI data was performed with SPM8. For each participant, we applied realignment (head motion correction), normalisation, coregistration (of the functional images to the T1-weighted images), and smoothing. In brief, images were realigned using rigid body transformations (29) to the first scan to correct for head motion ( 3 translation and 3 rotation parameters), followed by slicetiming correction by temporally aligning all slices to the same reference time point (first slice). We chose to perform realignment first to minimize the effect of inter-slice movement (30). Data were then normalized to the Montreal Neurological Institute (MNI) brain template. The normalized functional images were smoothed with an isotropic Gaussian kernel of $8 \mathrm{~mm}$ with full width at half maximum. FC by Independent Component Analysis (ICA)

FMRI data were decomposed into intrinsic functional networks using spatial ICA using the GIFT toolbox V3.0a (http://mialab.mrn.org/software/gift). Spatial ICA applied to fMRI data aims to separate spatially independent patterns from their linearly mixed BOLD signals via maximization of mutual independence among components (31). We limited the number of independent components (ICs) to 25 , similar to previous painrelated FC studies $(25,32,33)$. ICA is capable of extracting scanner or physiological 
noise and motion artefacts from the dataset (34), i.e. all non-neuronal ICs (e.g. respiration or head motion) were removed. To validate the stability of the resultant ICs, we employed ICASSO (35) (100 runs with random initiation) on the pre-processed data matrix for our model order of $k=25$ (ICS). In doing so, 1 IC from was removed from subsequent analysis, as the stability Index $l q$ was below 0.8 (out of 1 ). From the pool of remaining neuronal ICs (estimated across participants), we selected functionally relevant ICs using spatial correlation with previously defined templates, so that the final set of ICs consisted of the following (pain-related) networks: DMN, FPN, SN, SMN, posterior DMN (precuneus and posterior cingulate), anterior DMN (medial prefrontal cortex), inferior temporal, and visual network. Additionally, it was ensured that all remaining ICs had a similarity below $0.2(1=$ identical), which estimated from a cluster merging similarity dendrogram to minimize the chance of overlapping networks. Similar networks have been described in a study using a model of sustained deep tissue pain (32). Group ICA differences were reported at $p<0.05$ (using Bonferroni correction for multiple comparisons). We also extracted network-based FC strength (using GIFT) and correlated this parameter with structural markers. For simplicity, we focus on FC-FA interactions.FC by seed-to-whole-brain analysis

Seed-to-whole-brain FC (bandwidth $0.01-0.1 \mathrm{~Hz}$ ) was examined by spatiotemporal cross-correlations using CONN toolbox (v13o, http://www.nitrc.org/projects/conn/). 
WM, cerebrospinal fluid (CSF), and the six motion parameters were used as covariates of no interest. Only the WM and CSF signals were removed to avoid any bias introduced by removing the global signal (i.e., GM) $(36,37)$. This approach should 'normalize' the distribution of voxel-to-voxel connectivity values as effectively as including the global signal as a covariate of no interest, but without the potential problems of the latter method. Although we did not record respiration and cardiac responses, it has been demonstrated that non-neuronal physiological noise (e.g., cardiac and respiratory signal) can successfully be removed by the "CompCor" algorithm (36), as implemented in the Conn toolbox. As centrally involved in pain processing, the seed was placed in the mid-PAG region (MNI coordinates: $x=-1, y=-$ 28 , and $z=-6)$. Similar MNI coordinates for the PAG have been used in another FC study in pain patients (17). We first estimated FC maps on the single subject level. For second-level analysis, we then applied a univariate ANOVA to test for the main effect of group. Between-group comparisons were then performed using post-hoc unpaired two-tailed t-tests. The statistical threshold was first set to $p<0.001$ (uncorrected, $t>$ 3.2), as used in other $\mathrm{fMRI}$ studies to pain $(38,39)$. We then applied an additional cluster extent correction of $k \geq 24$ voxels to correct for multiple comparisons using the False Discovery Rate (FDR) correction (40) in order to achieve $p<0.05$ (corrected). Patient-specific depression anxiety, depression, pain intensity (VAS), and headache 
frequency ( $\mathrm{MOH}$ only) values were linked to FC strength using Pearson's correlations. To limit the number of multiple comparisons, FC strength was first extracted from the PAG-related FC and then masked by the regions showing FC group differences for the contrasts: $\mathrm{MOH}>\mathrm{CN}, \mathrm{MOH}<\mathrm{CN}, \mathrm{MYO}>\mathrm{CN}$, and $\mathrm{MYO}<\mathrm{CN}$.

\section{Structural imaging}

The T1-weighted volume sequences of the whole brain were acquired using a threedimensional MPRAGE (Magnetization Prepared Rapid Gradient Echo) sequence (time of repetition (TR): $8.7 \mathrm{~ms}$, echo time (TE): $2.3 \mathrm{~ms}$, flip angle: $8.0^{\circ}$, voxel-size $0.86 \times 0.86$ $\times 1.0 \mathrm{~mm}$, axial slice orientation, matrix size $256 \times 256$ ) on a 3T Philips Achieva scanner (Philips Healthcare, Best, The Netherlands), equipped with an eight-channel head coil as used in previous studies $(10,11)$.

Diffusion tensor imaging (DTI)

A 32-directional DTI scan was performed in all participants with the following parameters: number of slices: 60, TR: 10'331 ms, TE: $55 \mathrm{~ms}$, field of view: 224 x 120 x 
224, duration: $7.1 \mathrm{~min}$, number of diffusion b0-values: 2, slice thickness: $2 \mathrm{~mm}$, gap: 0 $\mathrm{mm}$, voxel resolution: $3.6 \times 3.6 \mathrm{~mm}$, flip angle: $90^{\circ}$, and $b$-value $=1000 \mathrm{~s} / \mathrm{mm}^{2}$.

DTI pre-processing

DTI data were processed and analyzed using FMRIB Software Library (FSL) software 5.0.7 (Oxford Centre for Functional Magnetic Resonance Imaging of the Brain Software Library; http://www.fmrib.ox.ac.uk/fsl) according to the default TBSS pipeline. Diffusion data were corrected for eddy-currents using the FMRIB's Diffusion Toolbox (FDT). Brains were extracted from non-brain tissues using the "bet" procedure (41). FA and MD images were created using "dtifit"procedure by fitting a diffusion tensor model at each voxel.

DTI analysis and statistics

Voxelwise statistical analysis of the FA data was carried out using TBSS (42), implemented on FSL (43). All subjects' FA data were non-linearly registered to an FMRIB58_FA standard-space image (FMRIB Centre, University of Oxford, Department of Clinical Neurology, John Radcliffe Hospital, Headington, Oxford, UK; http://www.fmrib.ox.ak.uk/FMRIB58 FA.html) and nonlinear transformed into MNI space. The mean image of all aligned FA images was created and thinned to provide a 
skeletonized mean FA image, which represents the center of all tracts common to the group. A threshold of FA at 0.2 was used to exclude GM or CSF and control for high inter-individual variability. Each aligned FA image of all participants was then projected onto this skeleton and the resulting data fed into voxelwise cross-subject statistics. Similar steps were carried out using the "tbss_non_FA" script to obtain mean diffusivity (MD) images which were also projected onto the mean FA skeleton. A quality control was performed by visual inspection of all FA images. Voxelwise statistical analysis on FA and MD was performed via a permutation-based inference for nonparametric statistical thresholding (5000 permutations) using FSL's "randomize" tool and two-sample t-tests (CN versus $\mathrm{MON}, \mathrm{CN}$ versus $\mathrm{MYO}, \mathrm{MOH}$ versus $\mathrm{MYO}$ ). Age, gender and total intracranial volume (TIV) were included as "covariates of no interest" in all TBSS analyses to attenuate any potential confounding effect on the variable of interest. TIV was used as variable of no interest as TIV was shown to influence FA and MD (44). The resulting statistical maps were thresholded at $p<0.05$ (family-wise error (FWE) corrected) using the threshold-free cluster enhancement (TFCE) method (45). Results were also evaluated at a TFCE uncorrected statistical level $(p<0.001)$, as used in other TBSS-related DTI studies in headache that have applied the TFCE method (46, 47). Regions were labeled anatomically by mapping the statistical map to JHU-WM Tractography and JHU ICBM-DTI-81 WM labels atlases. The association between 
disease duration, pain intensity, HADS-anxiety (HADS-A) and depression (HADS-D) scores to FA and MD values (in clusters with significant between-group differences) was examined at $p<0.001$ (uncorrected). Further, we looked at interactions between FA, MD, and FC values using Pearson's correlations.

Statistical analysis on demographical and clinical data

Chi-square $\left(\chi^{2}\right)$ test was used to analyze categorical data (e.g. gender) whereas oneway analysis of variance (ANOVA) followed by Tukey's post-hoc comparisons was applied for continuous variables (e.g. age, headache duration and frequency, anxiety and depression scores). The level of statistical significance was set at $p<0.05$.

\section{Results}

Demographics and clinical characteristics

Data on demographics are provided in Table 1. HADS-A and HADS-D scores did not differ between $\mathrm{MOH}$ and MYO $(p>0.05)$ but between CN and MYO $(p<0.001$ both for HADS-A and HADS-D) and $\mathrm{CN}$ and $\mathrm{MOH}(p<0.001$ both for HADS-A and HADS-D). There was a significant difference between $\mathrm{MOH}$ and MYO in pain duration $(p=0.002)$. 
Pain intensity, assessed by VAS, did not differ during the MR examination between patients (MOH: $4.2 \pm 2.1$; MYO: $5.5 \pm 1.7 ; p=0.14$ ). All patients were in pain (VAS $>0$ ) during the MRI. Medication in $\mathrm{MOH}$ and $\mathrm{MYO}$ is summarized in Table 2. All patients with $\mathrm{MOH}$ took triptans to treat their migraine attacks. Eleven $\mathrm{MOH}$ patients had migraine without aura, and 1 migraine with aura. In the MYO group 2 patients had episodic tension-type headache and 1 patient episodic migraine without aura.

fMRI: independent-component analysis

Head motion parameters during $\mathrm{fMRI}$ did not differ between groups with respect to rotation and translation values (all p's $>0.1$, unpaired two-tailed t-tests). In none of the examined groups, total displacement exceeded $1 \mathrm{~mm}$ or $1^{\circ}$, respectively, along the fMRI run. Hence, all subjects could be included in the subsequent FC analyses. We first applied the ICA to examine group differences on the network level (Figure 1 and Table 3). As shown in Figure 1, significant FC increases were seen in the $\mathrm{SN}(\mathrm{MOH}>\mathrm{CN}$ and $\mathrm{MOH}>\mathrm{MYO}$ ) and connected inferior temporal network ( $\mathrm{MOH}>\mathrm{CN}, \mathrm{MOH}>\mathrm{MYO})$. In contrast, significant FC decreases $(\mathrm{MOH}<\mathrm{CN}$ and $\mathrm{MYO}<\mathrm{CN})$ were seen in the bilateral FPN (including the PAG). 
fMRI: Seed-to-voxel analysis (seed: PAG)

The within-group seed-to-voxel analysis (Figure 2) showed that the CN group demonstrated FC between the PAG and dorsal ACC (label A), thalamus, posterior insula and visual areas, similar to recent FC studies using the PAG as seed region $(17,19)$. FC between PAG and the supplementary motor area (SMA, label B) was detected in both the $\mathrm{CN}$ and MYO groups, which was not significant in $\mathrm{MOH}$. In contrast, the $\mathrm{MOH}$ group exhibited FC of the PAG with the parietal cortex (including the precuneus and posterior cingulate cortex (label C), and rostral anterior cingulate cortex (ACC, label D). Further, both patient groups as well as the $\mathrm{CN}$ group showed FC between the PAG and surrounding midbrain regions. Between group differences are summarized in Table 4, Figures 3 and 4. Both patient groups showed higher FC between the PAG and left inferior frontal gyrus (IFG) and left cerebellar regions than CN (Figure 4 B and D). MOH had lower FC to regions including the visual cortex, precuneus and temporal regions and higher FC to precentral regions and OFC. In contrast, MYO had decreased FC to pre- and postcentral regions compared to $\mathrm{CN}$ and $\mathrm{MOH}$ (Figures 3 and 4). The direct comparison of both patients groups (Figure 4) revealed significant differences for the contrast $\mathrm{MOH}>\mathrm{MYO}$ only, with higher PAG-mediated FC to the pre- and postcentral 
gyri (BA 3/4/6, Figure 4, label 1), left subgenual ACC/caudate nucleus (Figure 4, label 2), and the right temporo-parietal junction (TPJ) (Figure 4, label 3).

Correlation of FC strength with clinical parameters

No significant correlations were found between (PAG mediated) FC strength and clinical values on $p<0.001$ (uncorrected).

DTI: Whole-brain WM analysis

No significant differences were found on total mean FA across groups (CN [mean, SD]: 0.335, 0.011; MOH [mean, SD]: 0.334, 0.012; MYO [mean, SD]: 0.335, 0.009; $p=0.999)$ and $\mathrm{MD}$ (CN [mean, SD]: $0.513 \times 10^{-3} \mathrm{~mm}^{2} / \mathrm{s}, 0.013 \times 10^{-3} \mathrm{~mm}^{2} / \mathrm{s} ; \mathrm{MOH}$ [mean, SD]: $0.507 \times 10^{-3} \mathrm{~mm}^{2} / \mathrm{s}, 0.015 \times 10^{-3} \mathrm{~mm}^{2} / \mathrm{s}, \mathrm{MYO}$ [mean, SD]: $0.512 \times 10^{-3} \mathrm{~mm}^{2} / \mathrm{s}, 0.012$ $\times 10^{-3} \mathrm{~mm}^{2} / \mathrm{s} ; p=0.611$ ). No significant group differences were detected at $p<0.05$, corrected with FWE. Significant group differences $(p<0.001$, uncorrected) are reported in Table 5. Compared with $\mathrm{CN}, \mathrm{MOH}$ had significantly reduced $\mathrm{FA}$ in the right parietal operculum (OP, Figure 5A, bottom panel) and increased FA in the left insula compared to $\mathrm{CN}$ (Figure 5A, top panel), On the other hand, patients with MYO showed increased FA in right insula relative to $\mathrm{CN}$ (posterior part, Figure 5A, top panel).

MD was significantly decreased in $\mathrm{MOH}$ compared to $\mathrm{CN}$ in the left insula (Figure 5B, top panel), There were no significant changes in MD in MYO (relative to $\mathrm{CN}$ ). The 
comparison 'MOH versus MYO' did not reveal any significant differences in FA values. Decreased MD values were found in $\mathrm{MOH}$ relative to MYO in several clusters located cerebellum (mostly right inferior and middle cerebellar peduncle and adjacent pontomedullary area), left lingual gyrus, pallidum, and fusiform gyrus (Table 5).

\section{DTI: Correlation of DTI with clinical parameters}

We then examined the correlations of FA and MD values with clinical scores. After controlling for age, gender and TIV within $\mathrm{MOH}$ group, we found no significant correlation, which survived $p<0.001$ (uncorrected).

DTI-fMRI: Correlation of network-based FC with FA

We next calculated if group FC differences (e.g. $\mathrm{MOH}>\mathrm{CN}$ ) were linked to group differences in FA. We found that FC of the SN was paralleled by higher FA values of the insular cortex for $\mathrm{MOH}>\mathrm{CN}$ (right insular cortex, $r=0.86, p<0.001$, Figure 6; left insular cortex: $r=0.58, p=0.008$, not shown) and across all patients (right insular cortex: $r=0.43, p=0.04$, results not shown).

\section{Discussion}


In the present study we found changes in FC and WM integrity in patients with $\mathrm{MOH}$ and MYO. On the network level, hypoconnectivity in the FPN was detected in both pain groups. In contrast, hyperconnectivity within the SN and inferior temporal network were features only in $\mathrm{MOH}$ (relative to $\mathrm{CN}$ and $\mathrm{MOH}$ ). Both pain groups had increased FC of the midbrain PAG with lateral frontal and cerebellar regions, whereas hyperconnectivity with orbitofrontal regions was only found in $\mathrm{MOH}$. In contrast, MYO had lower FC between PAG and sensorimotor cortex. Using DTI, increased FA was seen in the insula (both pain groups) and decreased FA in right OP (MOH only). MD changes were found in the left insula in $\mathrm{MOH}$. In $\mathrm{MOH}$, insular WM changes correlated with FC of the SN.

Our findings provide evidence that $\mathrm{FC}$ and $\mathrm{WM}$ alterations in $\mathrm{MOH}$ cannot be solely attributed to neuronal processes linked to chronic pain.

Functional connectivity: ICA results

FC alterations (relative to $\mathrm{CN}$ ) have been reported in migraine patients without aura (27), $\mathrm{MOH}(16)$, and fibromyalgia $(24,25)$. In the study by Russo et al. (27) FC was lower in the right FPN (= executive-working memory network) in patients with migraine without aura, including the middle frontal gyrus and dorsal ACC. In our study we found bilateral hypoconnectivity in the FPN in both pain ( $\mathrm{MOH}$ and $\mathrm{MYO}$ ) groups 
compared to $\mathrm{CN}$, suggesting a disturbed functionality of the executive network in chronic pain disorders, which is consistent with the notion of impaired executive (and memory) function in chronic pain (50). However, executive function was normal in patients with episodic migraine without aura (27).

Further we observed disturbed, saliency-related, functional communication in $\mathrm{MOH}$, reflected by hyperconnectivity within the $\mathrm{SN}$ for $\mathrm{MOH}$ relative to $\mathrm{CN}$ and $\mathrm{MYO}$. In addition, in the left insula we found increased FA and decreased MD (see below) in $\mathrm{MOH}$. In $\mathrm{MOH}, \mathrm{FC}$ strength of the SN was associated with FA in the insular cortex, suggesting that hyperconnectivity could ultimately lead to structural changes in parts of the SN, at least in the insular cortex. The insula and ACC are key structures within pain processing systems and have been related to attentional and perceptive aspects of pain (51). The ACC is involved in descending pain modulation, being functionally and anatomically connected with the PAG $(18,19)$. A previous study found increased FC between ACC and anterior insula in high frequency compared to low frequency migraine (52). Morphometric studies found decreased GM in the bilateral insula in $\mathrm{MOH}$, not reversible after detoxification $(10,11)$ and decreased cortical thickness in cingulate cortex and insula in high frequency vs. low frequency migraine (52). Hence consistent functional and structural alterations within the SN are demonstrated in $\mathrm{MOH}$. In contrast, a recent study on episodic migraine without aura reported 
decreased interhemispheric FC in the ACC and decreased FA in the corpus callosum $(52,53)$. In our study, MYO did not show significantly different FC relative to $\mathrm{CN}$ in the saliency regions, which was unexpected, as increased FC between insula and ACC has been reported in chronic pain (24).

Nevertheless, our results are in agreement to studies demonstrating a link between FC strength and structural markers of neuronal integrity such as FA $(54,55)$.

Functional connectivity: Seed-based results

The investigation of PAG-mediated FC analysis was motivated by the observation that the PAG shows (a) reversible structural alterations in $\mathrm{MOH}(10,11)$ and (b) has disturbed FC to several brain areas within nociceptive and somatosensory processing pathways in episodic migraine (17). The PAG is organized in distinct longitudinal columns that receive selective input from forebrain regions including OFC and cingulate cortex as well as sensory neurons from trigeminal nucleus and dorsal horn (18). It is a substantial component of the descending pain modulatory network, and exerts an inhibitory or excitatory control on nociceptive input via the rostral ventromedial medulla, which in turn projects to spinal and medullary dorsal horns (56). In the present study only one midbrain seed including right and left ventrolateral 
PAG was selected, because the FC between them was very high, consistent with previous studies $(17,19)$. Both patient groups showed increased FC between the PAG and left frontal and cerebellar regions, which suggests an association with chronic pain states rather than a pattern specific for $\mathrm{MOH}$. The dorsolateral prefrontal cortex is considered a part of pain processing matrices (51). The cerebellum is often reported in studies on chronic pain, and is functionally connected to the PAG $(19,57)$. In addition, PET studies reported hypermetabolic activity of the cerebellum in patients $\mathrm{MOH}$ before medication withdrawal (9).

Patients with $\mathrm{MOH}$ had a distinct pattern of $\mathrm{FC}$ between PAG (relative to MYO) in several regions implicated in the pathophysiology of migraine and $\mathrm{MOH}$ : Stronger FC to the bilateral OFC and weaker FC to occipital and mid cingulate regions. The OFC shows hypometabolism in $\mathrm{MOH}$ that persisted after withdrawal and GM volume in OFC predicted treatment response $(9,11)$. Dysfunction of OFC has been implicated in maladaptive decision making in dependence related disorders, and $\mathrm{MOH}$ has been discussed in this context (58).

Cortical spreading depression (CSD), the pathophysiological correlate of migraine aura, starts in visual cortex (59). Clinically "silent" CSDs probably occur in migraine without aura and the vulnerability for recurrent CSDs was found increased in an animal model 
of $\mathrm{MOH}(20,60)$. Decreased connectivity between PAG and occipito-parietal regions might reflect dysfunction of these regions with increased susceptibility for CSD and disturbed interplay with pain modulation in the brainstem.

In MYO, connectivity between PAG and SMN was decreased. Alterations in resting state activity in the SMN have been described in a model of myofascial pain. Specifically, connectivity of the SMN is disrupted after continuous painful stimulation, and shifted to the SN (32).

Comparing PAG-related connectivity between $\mathrm{MOH}$ and $\mathrm{MYO}$, we found decreased FC to the SMN in MYO, similar as in the comparison with controls. In addition, we observed increased FC between the PAG and the right TPJ and left subgenual ACC in $\mathrm{MOH}$ compared to MYO. The right TPJ is involved in the processing of social interactions and in the empathy for pain $(61,62)$. The subgenual ACC - which projects to the ventromedial striatum and the accumbens area - is involved in reward processing and addiction $(63,64)$. We conclude that these $\mathrm{FC}$ increases in $\mathrm{MOH}$ may be the result of a disturbed interplay between nociceptive and cognitive control brain regions.

DTI results 
WM changes in patients with chronic pain conditions might occur due to both (progressive) maladaptive plasticity over pain chronic manifestation (65). The notion of maladaptive plasticity in pain regions is in line with our findings of both increased FA in the insular cortex in $\mathrm{MOH}$ and $\mathrm{MYO}$, as well as decreased $\mathrm{FA}$ in right parietal operculum ( $\mathrm{MOH}$ only). Several factors may contribute to decreased FA, including increased branching, more crossing fibers or axons, edema, changes to protein filaments, cell membrane disruption and/or decreased myelin. Prolonged nociceptive activity may be related to central sensitization that contributes to chronic pain manifestation and the initiation of central neuroinflammatory processes $(66,67)$.

It is known that the $\mathrm{OP}$, posterior insula (and cingulate cortex) receive a large proportion of the spinothalamic cortical input in primates via the central ascending nociceptive pathway and have been implicated in response to painful stimulation (51). Group differences $(\mathrm{MOH}<\mathrm{CN})$ in the $\mathrm{OP}$ region are hence fully in line to recent interpretation of the medial and parietal opercular regions as somatosensory regions devoted to the processing of spinothalamic inputs $(51,68)$.

The insula has been implicated in the perception of pain (51) both in acute and chronic pain conditions. From a functional point of view, posterior and mid insular cortex are predominantly associated with the sensory aspect of pain (69-71), whereas anterior 
insula has been implicated to the cognitive-affective dimension of pain which is sensitive to contextual manipulations $(72,73)$. A recent combined structural and FC analysis of insular subdivisions (74) further supported that anterior insular part is predominantly connected to (pre)frontal regions (e.g. OFC and ventrolateral prefrontal), whilst the posterior insular part is strongly connected to somatosensory regions. Mid insula integrates sensory and cognitive emotional information (75) and displays widespread connections with parietal and temporal regions, the IFG, OFC and premotor cortex (76). According to the same insular subdivision system, we found increased $\mathrm{FA}$ in the mid insular part in $\mathrm{MOH}$ and in right posterior insular part in patients with MYO.

\section{Limitations of the study}

In contrast to previous studies on episodic migraine, patients with $\mathrm{MOH}$ were not scanned in pain free intervals but experienced headaches during scans. This approach was chosen, as most patients had almost daily headaches. The group MYO was also not pain free during scans. Also, abstinence from acute pain medication was not requested, as this might induce acute withdrawal headaches. An influence of chronic medication use on FC and DTI measures (such as FA and MD) is unknown to date but 
cannot be fully excluded. Pain duration differed between patient groups. As pain duration was not associated with changes in FC or WM integrity an effect on group comparisons was considered unlikely.

DTI findings are reported at $p<0.001$ uncorrected (for multiple comparisons), as the sample size was rather small, similar as in previous studies $(46,47)$. However, structural and functional changes overlapped in the insula showing a significant correlation between functional and structural measures. All statistical comparisons for fMRI survived a threshold of $p<0.05$ (corrected), which underlines the robustness of these findings.

\section{Interpretation}

Chronic pain may be associated with changes in resting state FC and white matter tracts, independent from the underlying etiology. Hyperconnectivity (relative to controls) within the saliency network along with associated white matter changes therein suggest a particular role of this network in $\mathrm{MOH}$. Further, in $\mathrm{MOH}$ regions related to internal control, pain modulation and CSD showed impaired connectivity with the PAG, probably related to an imbalance between descending pain facilitation/inhibition. 


\section{Acknowledgements}

This work was supported by a grant (DFL_MF 1149) of the Centre for Clinical research, University and University Hospital Zurich to author Franz Riederer. Foteini Christidi is supported by the State Scholarships Foundation (I.K.Y.), the Action "State Scholarships Foundation's mobility grants programme for the short term training in recognized scientific / research centres abroad for candidate doctoral or postdoctoral researchers in Greek universities or research centres", and the "IKY Fellowships of Excellence for Post-Graduate Studies in Greece-Siemens Program".

\section{Figure legends}

Figure 1. Summary of the functional connectivity $(\mathrm{FC})$ results, using an independent component analysis (ICA). Statistical group comparisons are shown in a color code 
(white bar: $p>0.05$, green bar: $p<0.05$ (using Bonferroni correction for multiple comparisons). $\mathrm{CN}$ : healthy controls, $\mathrm{MOH}$ : medication overuse headache, MYO: myofascial pain.

Figure 2. Within-group seed-to-voxel results. The seed was placed in in the PAG (MNI coordinates: $-1,-28,-6)$. Letters correspond to the following brain regions: $A=$ dorsal anterior cingulate cortex, $\mathrm{B}=$ supplementary motor area, $\mathrm{C}=$ posterior cingulate cortex and adjacent (posteriorly located) precuneus, $\mathrm{D}=$ rostral anterior cingulate cortex. The statistical threshold is set to $p<0.05$ (FDR corrected $t>3.2$ ). CN: healthy controls, $\mathrm{MOH}$ : medication overuse headache, MYO: myofascial pain.

Figure 3. Seed-to-voxel results comparing healthy to pain groups. The seed was placed in centrally in the PAG (MNI coordinates: $-1,-28,-6)$. Labels indicate different brain regions. The statistical threshold is set to $p<0.05$ (FDR corrected, $\mathrm{t}>3.2$ ). $\mathrm{MOH}$ : medication overuse headache, MYO: myofascial pain, CN: healthy controls.

Figure 4. Seed-to-voxel results comparing medication overuse pain $(\mathrm{MOH})$ to myofascial (MYO) pain. The seed was placed in centrally in the PAG (MNI coordinates: $1,-28,-6)$. Labels indicate different brain regions. The statistical threshold for $\mathrm{MOH}>$ 
MYO is set to $p<0.05$ (FDR corrected, $t>3.2$ ). 1: caudate nucleus, 2: postcentral gyrus, and 3: right temporo-parietal junction.

Figure 5. Summary of the diffusion tensor imaging (DTI) group results. Displayed are the group differences between $\mathrm{CN}$ and $\mathrm{MOH}, \mathrm{CN}$ and MYO. Statistical threshold is set to $p<0.001$ (uncorrected)). CN: healthy controls, $\mathrm{MOH}$ : medication overuse headache, MYO: myofascial pain.(A) Differences in functional anisotropy (FA) of the right insular cortex and parietal operculum (OP) for the contrast $\mathrm{MOH}-\mathrm{CN}$ and $\mathrm{MYO}-\mathrm{CN}$. (B) Differences in mean diffusivity (MD) of the left insular cortex and posterior cingulum for the contrast $\mathrm{MOH}-\mathrm{CN}$ and $\mathrm{MYO}-\mathrm{CN}$.

Figure 6. Interaction between functional connectivity (FC) strength and structural (neuronal) integrity. In $\mathrm{MOH}, \mathrm{FC}$ of the SN was positively linked to functional anisotropy (FA) of the right $(A)$ and left $(B)$ insular cortex at $p<0.001$. 


\section{Table legends}

Table 1: Demographics and clinical characteristics. Groups did not differ with respect to age or gender. Anxiety (HADS-A) and depression scores (HADS-D) were not different between $\mathrm{MOH}$ and $\mathrm{MYO} .{ }^{a} \mathrm{HC}$ vs. $\mathrm{MOH} ; \mathrm{MOH}$ vs. $\mathrm{MYO},{ }^{b} \mathrm{HC}$ vs. $\mathrm{MOH} ; \mathrm{HC}$ vs. MYO; $\mathrm{MOH}$ vs. MYO, ${ }^{\mathrm{C}} \mathrm{HC}$ vs. $\mathrm{MOH} ; \mathrm{HC}$ vs. MYO. HADS-A: anxiety. HADS-D: depression. $\mathrm{CN}$ : healthy controls, MOH: medication overuse headache, MYO: myofascial pain.

Table 2: Medication dosages for the $\mathrm{MYO}$ and $\mathrm{MOH}$ groups. $\mathrm{MOH}$ : medication overuse headache, MYO: myofascial pain.

Table 3: ICA results. Significant group differences are indicated by ${ }^{*}(p<0.05$, using Bonferroni correction for multiple comparisons). $\mathrm{CN}$ : healthy controls, $\mathrm{MOH}$ : medication overuse headache, MYO: myofascial pain. 
Table 4: Seed-to-voxel group differences. The seed was placed in the central PAG. All results are shown at $p<0.05$ (corrected for multiple comparisons using FDR correction). CN: healthy controls, $\mathrm{MOH}$ : medication overuse headache, MYO: myofascial pain. Table 5: DTI results. Results are displayed at $p<0.001$ (uncorrected). $\mathrm{CN}$ : healthy controls, $\mathrm{MOH}$ : medication overuse headache, MYO: myofascial pain.

\section{Key Findings}

- Functional connectivity identifies different networks in $\mathrm{MOH}$ and $\mathrm{MYO}$

- $\mathrm{MOH}$ and MYO demonstrate lower functional connectivity in the fronto-parietal attention network than controls

- $\mathrm{MOH}$ show increased functional connectivity in the saliency network

- Diffusion tensor imaging indicates disturbed white matter integrity in the insular cortex in $\mathrm{MOH}$ and $\mathrm{MYO}$ as well as in the parietal operculum ( $\mathrm{MOH}$ only)

Functional connectivity strength of the saliency network was positively correlated to structural neuronal integrity of the insular cortex in $\mathrm{MOH}$ 


\section{References}

[1] Diener HC, Limmroth V. Medication-overuse headache: a worldwide problem. The Lancet Neurology. 2004;3(2004):475-83.

[2] Evers S, Marziniak M. Clinical features, pathophysiology, and treatment of medicationoveruse headache. Lancet Neurol. 2010;9(2010):391-401.

[3] Headache Classification Committee of the International Headache S. The International Classification of Headache Disorders, 3rd edition (beta version). Cephalalgia : an international journal of headache. 2013;33(2013):629-808.

[4] De Felice M, Ossipov MH, Wang R, Lai J, Chichorro J, Meng I, et al. Triptan-induced latent sensitization: a possible basis for medication overuse headache. Annals of neurology. 2010;67(2010):325-37.

[5] Munksgaard SB, Bendtsen L, Jensen RH. Modulation of central sensitisation by detoxification in $\mathrm{MOH}$ : results of a 12-month detoxification study. Cephalalgia : an international journal of headache. 2013;33(2013):444-53.

[6] Okada-Ogawa A, Porreca F, Meng ID. Sustained morphine-induced sensitization and loss of diffuse noxious inhibitory controls in dura-sensitive medullary dorsal horn neurons. The Journal of neuroscience : the official journal of the Society for Neuroscience.

2009;29(2009):15828-35.

[7] Perrotta A, Serrao M, Sandrini G, Burstein R, Sances G, Rossi P, et al. Sensitisation of spinal cord pain processing in medication overuse headache involves supraspinal pain control. Cephalalgia : an international journal of headache. 2010;30(2010):272-84.

[8] Perrotta A, Arce-Leal N, Tassorelli C, Gasperi V, Sances G, Blandini F, et al. Acute Reduction of Anandamide-Hydrolase (FAAH) Activity is Coupled With a Reduction of Nociceptive Pathways Facilitation in Medication-Overuse Headache Subjects After Withdrawal Treatment. Headache. 2012;52(2012):1350-61.

[9] Fumal A, Laureys S, Di Clemente L, Boly M, Bohotin V, Vandenheede M, et al. Orbitofrontal cortex involvement in chronic analgesic-overuse headache evolving from episodic migraine. Brain : a journal of neurology. 2006;129(2006):543-50.

[10] Riederer F, Marti M, Luechinger R, Lanzenberger R, von Meyenburg J, Gantenbein AR, et al. Grey matter changes associated with medication-overuse headache: correlations with disease related disability and anxiety. The world journal of biological psychiatry : the official journal of the World Federation of Societies of Biological Psychiatry. 2012;13(2012):517-25. [11] Riederer F, Gantenbein AR, Marti M, Luechinger R, Kollias S, Sandor PS. Decrease of gray matter volume in the midbrain is associated with treatment response in medicationoveruse headache: possible influence of orbitofrontal cortex. The Journal of neuroscience : the official journal of the Society for Neuroscience. 2013;33(2013):15343-9.

[12] Egloff N, Sabbioni ME, Salathe C, Wiest R, Juengling FD. Nondermatomal somatosensory deficits in patients with chronic pain disorder: clinical findings and hypometabolic pattern in FDG-PET. Pain. 2009;145(2009):252-8. 
[13] Fox MD, Raichle ME. Spontaneous fluctuations in brain activity observed with functional magnetic resonance imaging. Nature reviews Neuroscience. 2007;8(2007):700-11. [14] Damoiseaux JS, Rombouts SA, Barkhof F, Scheltens P, Stam CJ, Smith SM, Beckmann CF. Consistent resting-state networks across healthy subjects. Proceedings of the National Academy of Sciences of the United States of America. 2006;103(2006):13848-53.

[15] Lee MH, Smyser CD, Shimony JS. Resting-state fMRI: a review of methods and clinical applications. AJNR American journal of neuroradiology. 2013;34(2013):1866-72.

[16] Chanraud S, Di Scala G, Dilharreguy B, Schoenen J, Allard M, Radat F. Brain functional connectivity and morphology changes in medication-overuse headache: Clue for dependencerelated processes? Cephalalgia : an international journal of headache. 2014;34(2014):605-15.

[17] Mainero C, Boshyan J, Hadjikhani N. Altered functional magnetic resonance imaging resting-state connectivity in periaqueductal gray networks in migraine. Annals of neurology. 2011;70(2011):838-45.

[18] Benarroch EE. Periaqueductal gray: an interface for behavioral control. Neurology. 2012;78(2012):210-7.

[19] Kong J, Tu PC, Zyloney C, Su TP. Intrinsic functional connectivity of the periaqueductal gray, a resting fMRI study. Behavioural brain research. 2010;211(2010):215-9.

[20] Pietrobon D, Striessnig J. Neurobiology of migraine. Nat Rev Neurosci. 2003;4(2003):386-98.

[21] Alexander AL, Lee JE, Lazar M, Field AS. Diffusion tensor imaging of the brain. Neurotherapeutics : the journal of the American Society for Experimental NeuroTherapeutics. 2007;4(2007):316-29.

[22] DaSilva AF, Granziera C, Tuch DS, Snyder J, Vincent M, Hadjikhani N. Interictal alterations of the trigeminal somatosensory pathway and periaqueductal gray matter in migraine. Neuroreport. 2007;18(2007):301-5.

[23] Granziera C, DaSilva AF, Snyder J, Tuch DS, Hadjikhani N. Anatomical alterations of the visual motion processing network in migraine with and without aura. PLoS Med.

2006;3(2006):e402.

[24] Cifre I, Sitges C, Fraiman D, Munoz MA, Balenzuela P, Gonzalez-Roldan A, et al. Disrupted functional connectivity of the pain network in fibromyalgia. Psychosomatic medicine. 2012;74(2012):55-62.

[25] Napadow V, LaCount L, Park K, As-Sanie S, Clauw DJ, Harris RE. Intrinsic brain connectivity in fibromyalgia is associated with chronic pain intensity. Arthritis and rheumatism. 2010;62(2010):2545-55.

[26] Gerwin RD. Classification, epidemiology, and natural history of myofascial pain syndrome. Current pain and headache reports. 2001;5(2001):412-20.

[27] Russo A, Tessitore A, Giordano A, Corbo D, Marcuccio L, De Stefano M, et al. Executive resting-state network connectivity in migraine without aura. Cephalalgia : an international journal of headache. 2012;32(2012):1041-8.

[28] Zigmond AS, Snaith RP. The hospital anxiety and depression scale. Acta Psychiatr Scand. 1983;67(1983):361-70. 
[29] Friston K, Ashburner J, Frith CD, Poline JB, Heather JD, Frackowiak RS. Spatial registration and normalization of images. Human brain mapping. 1995;3(1995):165-89.

[30] Sladky R, Friston KJ, Tröstl J, Cunnington R, Moser E, Windischberger C. Slice-timing effects and their correction in functional MRI. Neuroimage. 2011;58(2011):588-94.

[31] Stone JV. Independent component analysis: an introduction. Trends Cogn Sci. 2002;6(2002):59-64.

[32] Kim J, Loggia ML, Edwards RR, Wasan AD, Gollub RL, Napadow V. Sustained deeptissue pain alters functional brain connectivity. Pain. 2013;154(2013):1343-51.

[33] Napadow V, Kim J, Clauw DJ, Harris RE. Decreased intrinsic brain connectivity is associated with reduced clinical pain in fibromyalgia. Arthritis and rheumatism. 2012;64(2012):2398-403.

[34] Thomas CG, Harshman RA, Menon RS. Noise reduction in BOLD-based fMRI using component analysis. Neuroimage. 2002;17(2002):1521-37.

[35] Himberg J, Hyvarinen A, Esposito F. Validating the independent components of neuroimaging time series via clustering and visualization. Neuroimage. 2004;22(2004):1214-

22.

[36] Behzadi Y, Restom K, Liau J, Liu TT. A component based noise correction method (CompCor) for BOLD and perfusion based fMRI. Neurolmage. 2007;37(2007):90-101.

[37] Murphy K, Birn RM, Handwerker DA, Jones TB, Bandettini PA. The impact of global signal regression on resting state correlations: are anti-correlated networks introduced? Neurolmage. 2009;44(2009):893-905.

[38] Gimenez M, Pujol J, Ali Z, Lopez-Sola M, Contreras-Rodriguez O, Deus J, et al. Naproxen effects on brain response to painful pressure stimulation in patients with knee osteoarthritis: a double-blind, randomized, placebo-controlled, single-dose study. J Rheumatol.

2014;41(2014):2240-8.

[39] Smith JK, Humes DJ, Head KE, Bush D, White TP, Stevenson CM, et al. fMRI and MEG analysis of visceral pain in healthy volunteers. Neurogastroenterol Motil. 2011;23(2011):648e260.

[40] Benjamini Y, Hochberg Y. Controlling the False Discovery Rate - a Practical and Powerful Approach to Multiple Testing. Journal of the Royal Statistical Society Series BMethodological. 1995;57(1995):289-300.

[41] Smith SM. Fast robust automated brain extraction. Human brain mapping. 2002;17(2002):143-55.

[42] Smith SM, Jenkinson M, Johansen-Berg H, Rueckert D, Nichols TE, Mackay CE, et al. Tract-based spatial statistics: voxelwise analysis of multi-subject diffusion data. Neurolmage. 2006;31(2006):1487-505.

[43] Smith SM, Jenkinson M, Woolrich MW, Beckmann CF, Behrens TE, Johansen-Berg H, et al. Advances in functional and structural MR image analysis and implementation as FSL. Neurolmage. 2004;23 Suppl 1(2004):S208-19.

[44] Takao H, Hayashi N, Inano S, Ohtomo K. Effect of head size on diffusion tensor imaging. Neurolmage. 2011;57(2011):958-67. 
[45] Smith SM, Nichols TE. Threshold-free cluster enhancement: addressing problems of smoothing, threshold dependence and localisation in cluster inference. Neurolmage. 2009;44(2009):83-98.

[46] Absinta M, Rocca MA, Colombo B, Falini A, Comi G, Filippi M. Selective decreased grey matter volume of the pain-matrix network in cluster headache. Cephalalgia : an international journal of headache. 2012;32(2012):109-15.

[47] Neeb L, Bastian K, Villringer K, Gits HC, Israel H, Reuter U, Fiebach JB. No microstructural white matter alterations in chronic and episodic migraineurs: a case-control diffusion tensor magnetic resonance imaging study. Headache. 2015;55(2015):241-51.

[48] Nichols TE, Holmes AP. Nonparametric permutation tests for functional neuroimaging: a primer with examples. Human brain mapping. 2002;15(2002):1-25.

[49] Neeb L, Bastian K, Villringer K, Gits HC, Israel H, Reuter U, Fiebach JB. No Microstructural White Matter Alterations in Chronic and Episodic Migraineurs: A Case-Control Diffusion Tensor Magnetic Resonance Imaging Study. Headache. 2015(2015).

[50] Berryman C, Stanton TR, Bowering KJ, Tabor A, McFarlane A, Moseley GL. Do people with chronic pain have impaired executive function? A meta-analytical review. Clinical psychology review. 2014;34(2014):563-79.

[51] Garcia-Larrea L, Peyron R. Pain matrices and neuropathic pain matrices: a review. Pain. 2013;154 Suppl 1(2013):S29-43.

[52] Maleki N, Becerra L, Brawn J, Bigal M, Burstein R, Borsook D. Concurrent functional and structural cortical alterations in migraine. Cephalalgia : an international journal of headache. 2012;32(2012):607-20.

[53] Yuan K, Qin W, Liu P, Zhao L, Yu D, Zhao L, et al. Reduced fractional anisotropy of corpus callosum modulates inter-hemispheric resting state functional connectivity in migraine patients without aura. PloS one. 2012;7(2012):e45476.

[54] Supekar K, Uddin LQ, Prater K, Amin H, Greicius MD, Menon V. Development of functional and structural connectivity within the default mode network in young children. Neurolmage. 2010;52(2010):290-301.

[55] van Oort ESB, van Walsum AMV, Norris DG. An investigation into the functional and structural connectivity of the Default Mode Network. Neurolmage. 2014;90(2014):381-9.

[56] Heinricher MM, Tavares I, Leith JL, Lumb BM. Descending control of nociception: Specificity, recruitment and plasticity. Brain Res Rev. 2009;60(2009):214-25.

[57] Younger JW, Shen YF, Goddard G, Mackey SC. Chronic myofascial temporomandibular pain is associated with neural abnormalities in the trigeminal and limbic systems. Pain. 2010;149(2010):222-8.

[58] Fuh JL, Wang SJ, Lu SR, Juang KD. Does medication overuse headache represent a behavior of dependence? Pain. 2005;119(2005):49-55.

[59] Hadjikhani N, Sanchez Del Rio M, Wu O, Schwartz D, Bakker D, Fischl B, et al. Mechanisms of migraine aura revealed by functional MRI in human visual cortex. Proceedings of the National Academy of Sciences of the United States of America. 2001;98(2001):4687-92. 
[60] Green AL, Gu P, De Felice M, Dodick D, Ossipov MH, Porreca F. Increased susceptibility to cortical spreading depression in an animal model of medication-overuse headache.

Cephalalgia : an international journal of headache. 2013;34(2013):594-604.

[61] Decety J, Lamm C. The role of the right temporoparietal junction in social interaction: how low-level computational processes contribute to meta-cognition. Neuroscientist. 2007;13(2007):580-93.

[62] Akitsuki Y, Decety J. Social context and perceived agency affects empathy for pain: an event-related fMRI investigation. Neurolmage. 2009;47(2009):722-34.

[63] Drevets WC, Savitz J. The Subgenual Anterior Cingulate Cortex in Mood Disorders. Cns Spectrums. 2008;13(2008):663-81.

[64] Goldstein RZ, Volkow ND. Dysfunction of the prefrontal cortex in addiction: neuroimaging findings and clinical implications. Nature reviews Neuroscience.

2011;12(2011):652-69.

[65] Maleki N, Becerra L, Borsook D. Migraine: maladaptive brain responses to stress. Headache. 2012;52 Suppl 2(2012):102-6.

[66] Chiang CY, Dostrovsky JO, Iwata K, Sessle BJ. Role of glia in orofacial pain. The Neuroscientist : a review journal bringing neurobiology, neurology and psychiatry.

2011;17(2011):303-20.

[67] Latremoliere A, Woolf CJ. Central sensitization: a generator of pain hypersensitivity by central neural plasticity. The journal of pain : official journal of the American Pain Society. 2009;10(2009):895-926.

[68] Garcia-Larrea L. The posterior insular-opercular region and the search of a primary cortex for pain. Neurophysiologie clinique = Clinical neurophysiology. 2012;42(2012):299-313.

[69] Albanese MC, Duerden EG, Rainville P, Duncan GH. Memory traces of pain in human cortex. The Journal of neuroscience : the official journal of the Society for Neuroscience. 2007;27(2007):4612-20.

[70] Oertel BG, Preibisch C, Martin T, Walter C, Gamer M, Deichmann R, Lotsch J. Separating brain processing of pain from that of stimulus intensity. Human brain mapping. 2012;33(2012):883-94.

[71] Raij TT, Numminen J, Narvanen S, Hiltunen J, Hari R. Brain correlates of subjective reality of physically and psychologically induced pain. Proceedings of the National Academy of Sciences of the United States of America. 2005;102(2005):2147-51.

[72] Ploner M, Lee MC, Wiech K, Bingel U, Tracey I. Flexible cerebral connectivity patterns subserve contextual modulations of pain. Cerebral cortex. 2011;21(2011):719-26.

[73] Wiech K, Lin CS, Brodersen KH, Bingel U, Ploner M, Tracey I. Anterior insula integrates information about salience into perceptual decisions about pain. The Journal of neuroscience : the official journal of the Society for Neuroscience. 2010;30(2010):16324-31.

[74] Wiech K, Jbabdi S, Lin CS, Andersson J, Tracey I. Differential structural and resting state connectivity between insular subdivisions and other pain-related brain regions. Pain.

2014;155(2014):2047-55. 
[75] Craig AD. How do you feel--now? The anterior insula and human awareness. Nature reviews Neuroscience. 2009;10(2009):59-70.

[76] Cerliani L, Thomas RM, Jbabdi S, Siero JC, Nanetti L, Crippa A, et al. Probabilistic tractography recovers a rostrocaudal trajectory of connectivity variability in the human insular cortex. Human brain mapping. 2012;33(2012):2005-34.

[77] Radat F, Lanteri-Minet M. [Addictive behaviour in medication overuse headache: $A$ review of recent data]. Revue neurologique. 2011;167(2011):568-78.

[78] Saper JR, Hamel RL, Lake AE, 3rd. Medication overuse headache (MOH) is a biobehavioural disorder. Cephalalgia : an international journal of headache.

2005;25(2005):545-6. 